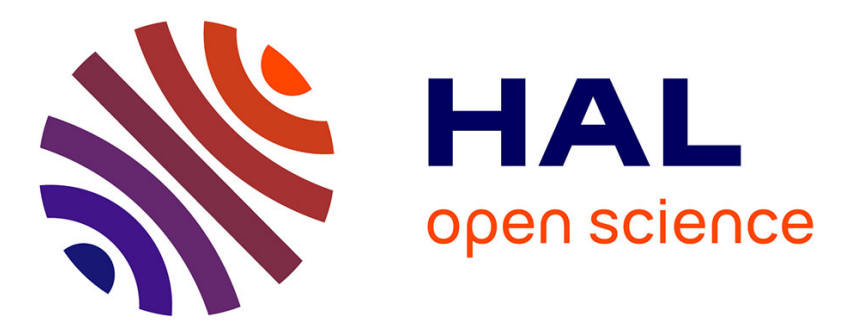

\title{
Comparative Assessment of a Novel Optical Human-Machine Interface for Laparoscopic Telesurgery
}

Fabien Despinoy, Luis Alonso Sanchez Secades, Nabil Zemiti, Pierre Jannin, Philippe Poignet

\section{- To cite this version:}

Fabien Despinoy, Luis Alonso Sanchez Secades, Nabil Zemiti, Pierre Jannin, Philippe Poignet. Comparative Assessment of a Novel Optical Human-Machine Interface for Laparoscopic Telesurgery. IPCAI: Information Processing in Computer-Assisted Interventions, Jun 2014, Fukuoka, Japan. pp.2130, 10.1007/978-3-319-07521-1_3. lirmm-01086444

\section{HAL Id: lirmm-01086444 https://hal-lirmm.ccsd.cnrs.fr/lirmm-01086444}

Submitted on 24 Nov 2014

HAL is a multi-disciplinary open access archive for the deposit and dissemination of scientific research documents, whether they are published or not. The documents may come from teaching and research institutions in France or abroad, or from public or private research centers.
L'archive ouverte pluridisciplinaire HAL, est destinée au dépôt et à la diffusion de documents scientifiques de niveau recherche, publiés ou non, émanant des établissements d'enseignement et de recherche français ou étrangers, des laboratoires publics ou privés. 


\title{
Comparative assessment of a novel optical human-machine interface for laparoscopic telesurgery
}

\author{
Fabien Despinoy ${ }^{1,2,3}$, Alonso Sánchez ${ }^{1}$, Nabil Zemiti ${ }^{1}$, \\ Pierre Jannin $^{2,3}$, and Philippe Poignet ${ }^{1}$ \\ 1 LIRMM - CNRS, UMR 5506, Université Montpellier 2, \\ Montpellier, F-34000, France \\ \{despinoy, sanchezsec, zemiti, poignet\}@lirmm.fr \\ 2 LTSI, Université de Rennes 1, Rennes, F-35000, France \\ ${ }^{3}$ INSERM, UMR 1099, Rennes, F-35000, France \\ pierre.jannin@univ-rennes1.fr
}

\begin{abstract}
This paper introduces a novel type of human-machine interface for laparoscopic telesurgery that employs an optical sensor. A Raven-II laparascopic robot (Applied Dexterity Inc) was teleoperated using two different human-machine interfaces, namely the Sigma 7 electromechanical device (Force Dimension Sarl) and the Leap Motion (Leap Motion Inc) infrared stereoscopic camera. Based on this hardware platform, a comparative study of both systems was performed through objective and subjective metrics, which were obtained from a population of 10 subjects. The participants were asked to perform a peg transferring task and to answer a questionnaire. Obtained results allow to confirm that fine tracking of the hand could be performed with the Leap Motion sensor. Such tracking comprises accurate finger motion acquisition to control the robot's laparoscopic instrument jaws. Furthermore, the observed performance of the optical interface proved to be comparable to that of traditional electro-mechanical devices, such as the Sigma 7, during adequate execution of highly-dexterous laparascopic gestures.
\end{abstract}

Keywords: Infrared stereoscopic camera, human-machine interface, teleoperation, laparoscopic surgery, hand tracking.

\section{Introduction}

During the last three decades, the field of laparoscopic surgery was constantly subject to technological advances looking to offer better healthcare in terms of safety, patient outcome, medical staff coordination and comfort [1]. Among these advances, the appearance of telesurgical systems represents a major breakthrough. Teleoperated systems enabled surgeons to interact with a distant environment, while providing them with a sense of immersion within the remote environment. To that end, high-resolution endoscopic cameras and intuitive humanmachine interfaces (HMIs) for controlling the robotized laparoscopic tools have 


\section{F. Despinoy et al.}

been developed. Moreover, it is well-known that telesurgical systems allow further performance improvements for surgeons by scaling down the hand motions (i.e. to perform more accurate gestures), filtering involuntary hand tremor and offering, in some cases, force-feedback information [2]. However, the lack of costeffective, sterilizable, precise and repeatable force sensing solutions represents an open issue, which is clearly reflected in currently available commercial robotic systems such as the Da Vinci ${ }^{\circledR}$ surgical system (Intuitive Surgical Inc) or the Raven-II platform [3] [4].

The majority of master-slave systems used in telesurgery exchange kinematic information of the operator's hands (e.g. through the system's position and/or velocity channels) in order to define the control reference of the robotic endeffectors [5]. Hence, the HMIs that are used to recover kinematic information from the surgeon do have a direct impact on the overall robotic system performance. For that reason, some recent research focused on the development of novel HMIs to improve dexterity and ergonomy. In [6], for instance, the authors presented an analysis of an electro-mechanical HMI for minimally invasive surgery, which is based on ergonomic principles and on polls carried out within the surgical community. Their results suggested that surgeons' preferences are mainly driven by 2 factors: comfort and precision. Nevertheless, the proposed electro-mechanical HMI is unable of fully exploiting the capabilities of the surgeon's hands. For example, their workspace is limited in order to avoid collisions between the left and right hand devices, whereas a human operator is capable of highly-dexterous and accurate bi-manual surgical manipulations within the frontal region that is unexploited by conventional electro-mechanical HMIs (e.g. the Sigma 7 employed in this work, or the Omega 7 modified in [6]). Therefore, ergonomy and intuitiveness of today's HMIs might be further improved.

In this paper, we address the issue of validating the possibility of performing highly-dexterous and precise telesurgical tasks by means of an infrared stereoscopic camera, the Leap Motion. Furthermore, initial results on the novel possibilities that could be offered by such type of interfaces are also introduced through a comparative study with a high-performance commercial electromechanical HMI, the Sigma 7.

Previous works employing optical based sensors in the context of teleoperation can be found in the literature. In [7], the Kinect device (Microsoft Corp) was employed to track the user's upper limbs and to control two industrial robot arms to perform pick and place tasks. Thanks to the anthropomorphic configuration of the robot arms, the user can easily control their motion by using joint angle inputs without major kinematic issues. Similarly, [8] implemented tracking algorithms to segment and detect the hand's thumb and index fingers in order to control the robot end-effector. However, their algorithms were highly sensitive to optical occlusions, and also relied on the detection of the entire forearm. Finally, whether a surgical application is considered, these approaches would fail to accurately distinguish finger motions for fine control of the instrument tips. In consequence, it might be more suitable to state that [7] and [8] employed less accurate hand gesture recognition techniques (e.g. open hand and 
fist gestures) for control of the robotic end-effectors. Finally, in [9] the authors performed simultaneous tracking of both hands and fingers in a robust manner, accounting for possible optical occlusions by estimating two 26 degrees of freedom (DoFs) hand models and performing optimization to find an unique fitted solution based on Kinect sensor measurements. Nevertheless, one major issue of their work regards its considerable computational complexity due to the solution of a high-order optimization problem using stochastic algorithms. Indeed, time delay and synchronization are two key factors which affect the performance of the entire teleoperation system. Therefore, a simplified and less computationally expensive solution using a 7 DoF hand model is introduced in this paper for tracking of the hand within the context of laparoscopic surgery.

The remaining sections are structured as follows. Section II introduces the teleoperation platform with the two different HMIs that were compared. Section III describes the methods employed for evaluating the performance of the system and the obtained results are also presented and analyzed. Lastly, in section IV concluding remarks and future works are discussed.

\section{The teleoperation setup}

In order to effectively compare the relative performance of both HMI technologies, the platform depicted in Figure 1 was employed. Such a scheme was proposed to isolate the influence of the HMIs by keeping constant the other hardware components (i.e master components and the slave robot system). For that reason, an unified HMI application programming interface (API), which allows quick exchange of the interfaces, was implemented by software wrapping of the corresponding device drivers. A footpedal was used in order to activate/deactivate teleoperation when required.

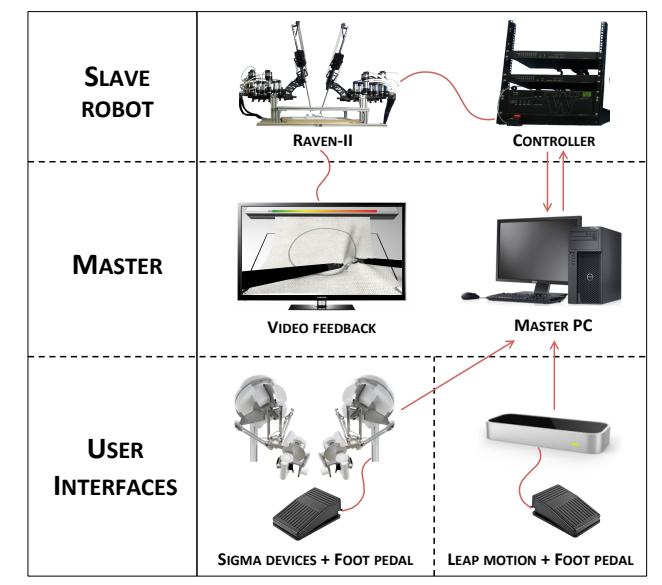

Fig. 1. Overall diagram of the teleoperation setup. 


\section{F. Despinoy et al.}

In Figure 2, pictures of the actual slave side system components are provided, including the peg board used for laparoscopic task evaluation. The Raven-II robot comprises two robotic arms of 7 DoFs, in which the two distal DoFs are decoupled and allow independent movements of each grasping jaw. During the experiments with both HMIs, the same Cartesian position servoing software routines were employed at a control frequency of $1 \mathrm{kHz}$.

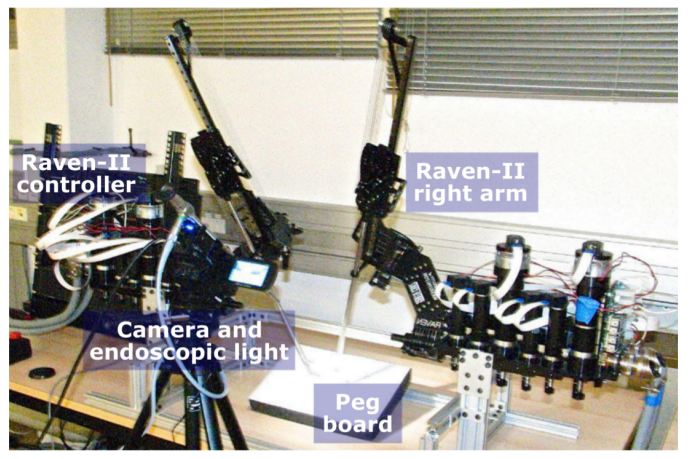

(a) Raven-II system

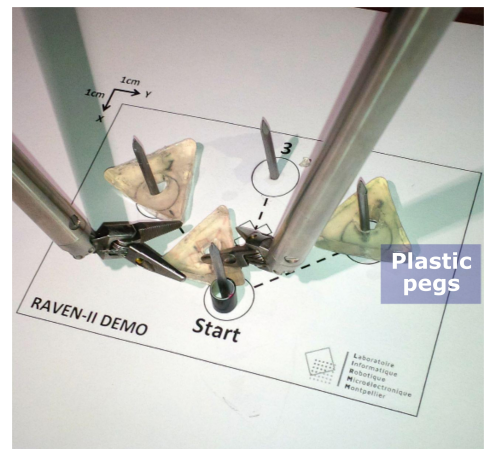

(b) Peg board

Fig. 2. Slave side hardware.

Figure 3 depicts the main master side system components. Each of the Sigma devices has 7 active DoFs. A Cartesian position measurement accuracy of about $0.005 \mathrm{~mm}$ (including translation and grasping motions) is reported by the manufacturer. During tests, these devices were polled at a $1 \mathrm{kHz}$ rate. Since the Sigma interfaces were disposed in order to avoid workspace overlapping of the left and right hands, no risk of collision between the HMIs exists.

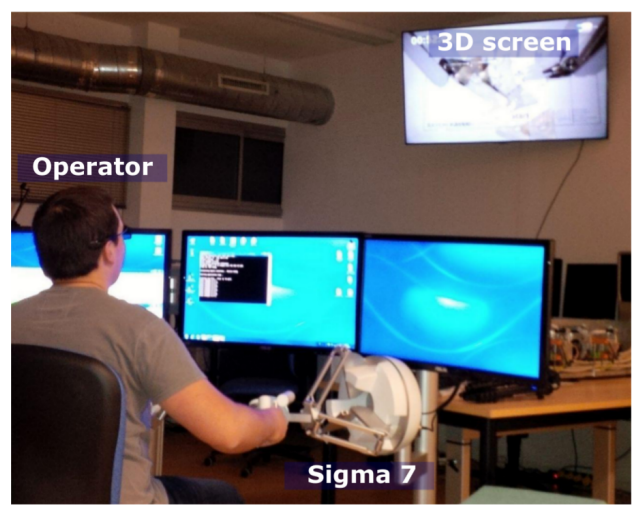

Fig. 3. Sigma 7 based master side hardware. 


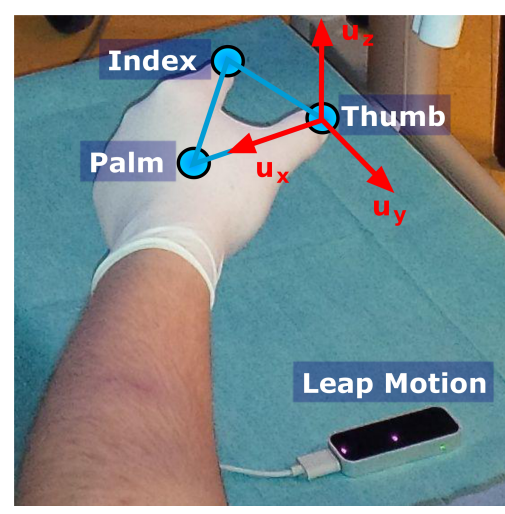

(a) Infrared stereoscopic camera

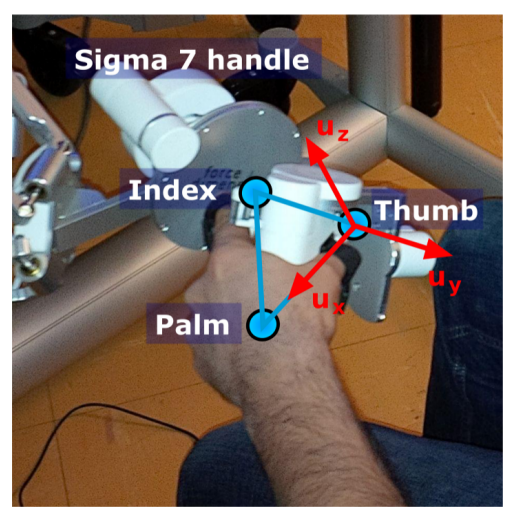

(b) Electro-mechanical device

Fig. 4. Hand model geometry shown for both HMIs.

Regarding the Leap Motion HMI, visible in Figure 4(a), three infrared sensors coupled with an array of two cameras is used to create a depth map of the scene. The Leap Motion's Cartesian position measurement accuracy is about $0.01 \mathrm{~mm}$, and the employed hand tracking refresh rates were $\approx 110 \mathrm{~Hz}$. The API provided by the manufacturer allows tracking of hand palms and fingers, the latter being classified as pointy objects. Such device could also allow left and right hand workspaces overlapping without the associated risks of HMI collisions. Such possibility might be advantageous during some laparascopic tasks (e.g. intracorporeal knotting and peg or needle transfers from one hand to another), which could otherwise turn to be less comfortable and intuitive. Nonetheless, whether the hand workspaces intersect, particular care should be taken when optical hand occlusions arise. For that reason, a simple model consisting of three points (i.e. index finger, thumb finger and hand palm) was defined in order to allow robust model based tracking using a simple time-consistent Kalman filter. The model geometry is depicted in Figures 4(a) and (b).

Tremor filtering is applied in both HMIs through an autoregressive movingaverage (ARMA) low-pass filter, having an attenuation of $\approx 25 \mathrm{~dB}$ at $2 \mathrm{~Hz}$ [10]. Finally, whenever hand tracking is lost, special care was taken in order to stop teleoperation (i.e. overriding the footpedal), while avoiding bumps when the tracking is recovered.

\section{Performance assessment of the HMIs}

\subsection{Methods}

A population of ten researchers from the LIRMM Lab were enrolled in the comparative performance evaluation of the HMIs. A video was shown to each candidate in order to introduce the overall system functioning and the demanded 
peg transferring sequence. The latter sequence consisted in transferring some pegs directly from one pin to another, and takes into account guidelines provided by SAGES and FLS organizations [11]. The sequence is defined as follows:

1. Pick the first peg with the left tool and insert it in target 1 (leftmost pin of Fig. 2(b))

2. Pick the second peg with the right tool and insert it in target 2 (rightmost pin)

3. Pick the latest peg with the left or right tool, then advance towards the center of the peg board to grab it with the other available tool in order to finally insert it in target 3 (uppermost pin)

Each subject began the protocol with a randomly selected HMI and was allowed to freely use the device during 5 minutes, allowing him/her to test-drive the teleoperated system, familiarize with movements and hand coordination. During this test-drive, the participant was taught how the system works, about its possibilities and limitations (i.e. avoiding prolonged-time occlusions when using the Leap Motion or engaging teleoperation at the workspace limits of the Sigma 7). Subsequently, the candidates were asked to repeat the presented peg transferring sequence five times with each device. The last three trials were used for the statistical analysis. Finally, the subjects were asked to grade each HMI through multiple measurements.

\subsection{Results}

Recorded data was analyzed using the JMP 11 software (SAS Institute Inc). A one-way analysis of variance (ANOVA) was carried out by defining the HMI device as a fixed factor and the duration as the response variable. ANOVA relies on the variance analysis from multiple samples to determine their affiliation and whether a difference between mean durations exists, and thus, to accept or discredit $H_{0}$ hypothesis, which means that each device allows to perform the same task with the same duration time. Before performing ANOVA calculation, we ensured that data respect a normality function for an homogeneous repartition (i.e. $\chi^{2}$ normality test).

Figure 5(a) depicts the average value and standard deviation of the total task completion time for all subject trials with each device. It is observed that the candidates were able to execute the task $13 \%$ faster in the case of the Sigma 7 device. ANOVA computation results are summarized in Figure 5(b), which shows the mean difference of duration times for each device. With a p-value of 0.0172 , this analysis highlights that a significant difference between both devices can be confirmed, so that they can constitute two distinct populations. Nevertheless, even though a difference is confirmed, there is only a small gap between both devices (i.e. 14 seconds for a task requiring $\approx 1$ min 50 secs).

Figure 6(a) shows the success rate of peg transfers. A fail was attributed to the operator whenever a peg was dropped before reaching the intended target. A 


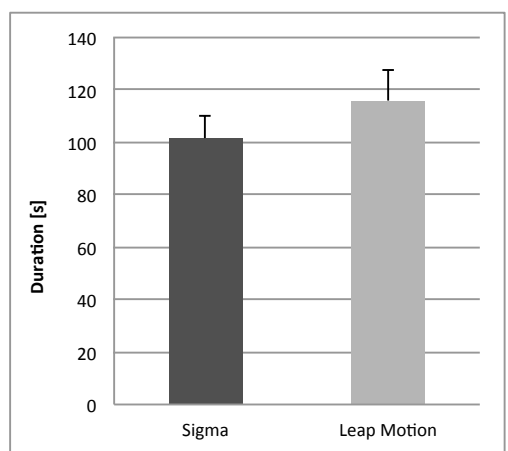

(a) Task completion time

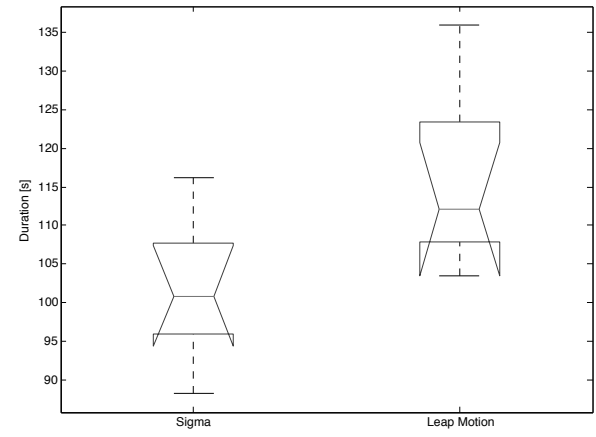

(b) One-way ANOVA

Fig. 5. Duration of the pick and place task for all subjects and repetitions, with standard deviations for each HMI. The one-way ANOVA analysis shows the median, first and third quartiles and highlights the significant mean duration difference of the HMIs based on the variance of each population $(p$-value $=0.0172)$.

higher success rate was observed in the case of the Leap Motion, signifying that, in spite of the slightly larger task completion times, the operators committed less mistakes with this device.

Similarly, the number of times the users had to clutch the teleoperation system through the footpedal was recorded (see "Clutching" column in Figure 6(b)). Subjects had to do this in order to reposition themselves for being able to carry on with the peg transferring task (e.g. hand tracking loss, reaching the workspace limits). It can be observed that better clutching results could be obtained with the Sigma 7 device, mainly due to the simple occlusions handling strategy that was implemented for the Leap Motion device. Consequently, operators were not able to properly detect the limit of the manipulation workspace, leading to a security lock and forced subjects to clutch in order to recover the hand tracking. Indeed, similar completion times, clutching and success rates can be obtained with both devices (see "Time", "Success" and "Clutching" columns in Figure $6(\mathrm{~b})$ ), whereas two additional metrics highlight a significant difference between the HMIs. On the one hand, the cost of each interface is taken into account (see "Cost" column in Figure 6(b)), in favor of the Leap Motion sensor. Even so, the high cost of the Sigma 7 interface is mainly explained by the force-feedback embedded technology which is not employed during the experimentations. Alternative positioning systems such as the MicroScribe ${ }^{\circledR}$ (Solution Technologies Inc) or the Phantom Omni ${ }^{\circledR}$ haptic device (Sensable, now part of Geomagic) can be taken on board for a more relevant comparison. Nevertheless, the Leap Motion device is still one of the cheaper interactive device present on the market. On the other hand, the sterilization capacity was compared (see "Sterilization" column in Figure 6(b)). This latter measurement refers to the possibility for the surgeon of preserving asepsis (i.e. hygiene without considering the certification requirements) during the whole duration of the procedure, so that he/she can directly 


\section{F. Despinoy et al.}

operate in the patient, if required, without forced to change surgical gloves. An upper grade was attributed to the Leap Motion device, since infrared light can easily traverse a thin film of plastic to prevent it from contaminating the sterile environment. In the case of the Sigma 7 , a lower grade was attributed to the device, since the presented system is not certified to meet such requirement.

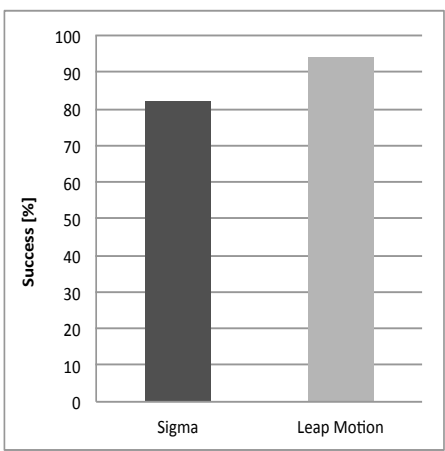

(a) Success rates

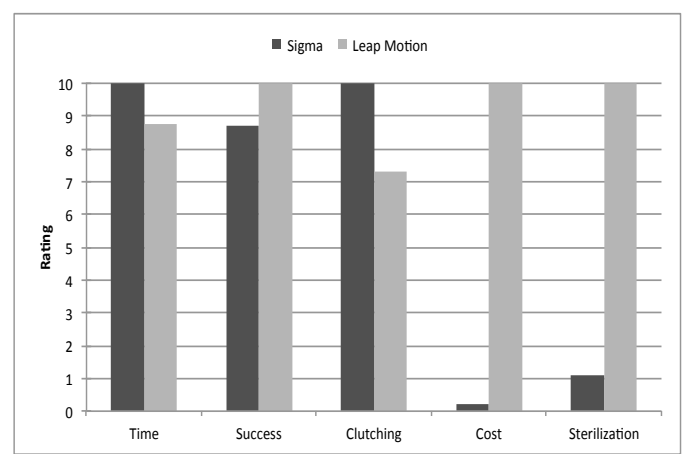

(b) Objective metrics

Fig. 6. Success of the pick and place task for all subjects and repetitions. Evaluation of the system through five normalized objective metrics.

Figure 7 summarizes the results that were obtained after questioning each operator. It can be confirmed that the Sigma 7 device outperformed the Leap Motion in terms of perceived reactivity, precision, robustness and comfort. Nevertheless, in terms of intuitiveness, the operators were more satisfied with the contact-less optical device. It is also observed that important ameliorations in terms of robustness are imperative for future experimentations, including an accurate optical occlusion management.

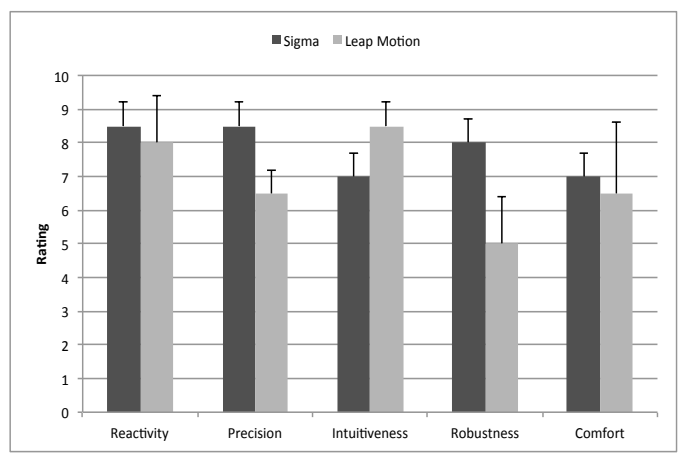

Fig. 7. Attributed grades, for each subjective factor, by all operators. 


\section{Conclusions and future works}

Considering the advantages and disadvantages of the Sigma 7 and the Leap Motion devices, together with their relative performance during the peg transferring task, our initial results suggest that infrared stereoscopic camera sensors have a promising potential for the development of cost-effective, sterilization compatible, more intuitive and accurate HMIs for laparoscopic telesurgery. Nonetheless, the obtained results also indicate that imperative development are required to improve the robustness of the hand tracking algorithms in the presence of optical occlusions.

Some design recommendations for next-generation HMIs could also be suggested from this first study. Report from experimentation highlights that the enlarged workspace and the freedom of bi-manual interactions between hands was appreciated by subjects. Furthermore, alternative solutions allowing forcereflecting teleoperation might be considered in the future, whether appropriate force sensing solutions for the medical field become available, through the development of wearable antagonistic tendon-like systems, exoskeletons, or through the application of model based electrical stimulation of the upper limbs.

Immediate future works mainly regard the development of a more robust model based hand tracking. Unfortunately, the API of the Leap Motion is closed by the device provider. Therefore, other specific sensors with open APIs, such as the Senz3D (Creative Labs Ltd, time-of-flight sensor), might be employed in the future. Additionally, a complementary study relying on trajectory analysis, from both master and slave sides, should be considered in order to enhance the interpretation of the results obtained during these experimentations.

Finally, other surgical tasks requiring higher accuracy, such as dissection of small targets, suturing and clip application should be studied, including expert and novice surgeon operators, in order to improve the statistical significance of our first results.

\section{Acknowledgements}

This work was partially supported by French state funds managed by the ANR within the Investissements d'Avenir programme (Labex CAMI) under reference ANR-11-LABX-0004.

\section{References}

1. Sanchez, A., Poignet, P., Dombre, E., Menciassi, A., Dario, P. : A Design Framework for Surgical Robots: Example of the ARAKnes Robot Controller. Journal of Robotics and Autonomous Systems, special issue on Intelligent Autonomous Systems. Lee, S., Lee, J.M. and Menegatti, E., editors. Elseiver, in press (2013) 


\section{F. Despinoy et al.}

2. Rosen, J., Hannaford, B., MacFarlane, M.P., Sinanan, M.N. : Force Controlled and Teleoperated Endoscopic Grasper for Minimally Invasive Surgery-Experimental Performance Evaluation. IEEE Transactions on Biomedical Engineering, 46(10), 1212-1221 (1999)

3. Lum, M.J.H., Friedman, D.C.W., Sankaranarayanan, G., King, H., Fodero, K., Leuschke, R., Hannaford, B., Rosen, J., Sinanan, M.N. : The raven: Design and Validation of a Telesurgery System. The International Journal of Robotics Research, 28(9), 1183-1197 (2009)

4. Hannaford, B., Rosen, J., Friedman, D.W., King, H., Roan, P., Cheng, L., Glozman, D., Ma, J., Kosari, S.N., White, L. : Raven-II: An Open Platform for Surgical Robotics Research. IEEE Transactions on Biomedical Engineering, 60(4), 954-959 (2013)

5. Lawrence, D.A. : Stability and Transparency in Bilateral Teleoperation. IEEE Transactions on Robotics and Automation, 9(5), 624-637 (1993)

6. Santos-Carreras, L., Hagen, M., Gassert, R., Bleuler, H. : Survey on Surgical Instrument Handle Design: Ergonomics and Acceptance. Journal of Surgical Innovation 19(1), 50-59 (2012)

7. Dragan, A.D., Srinivasa, S.S, Lee, K.C.T. : Teleoperation with Intelligent and Customizable Interfaces. Journal of Human-Robot Interaction 2(2), 33-57 (2013)

8. Du, G., Zhang, P., Mai, J., Li, Z. : Markerless Kinect-Based Hand Tracking for Robot Teleoperation. International Journal of Advanced Robotic Systems, 9(1), 36-46 (2012)

9. Oikonomidis, I., Kyriazis, N., Argyros, A.A. : Tracking the Articulated Motion of Two Strongly Interacting Hands. IEEE Conference of Computer Vision and Pattern Recognition, 1862-1869 (2012)

10. Bo, A.P.L., Poignet, P., Geny, C. : Pathological Tremor and Voluntary Motion Modeling and Online Estimation for Active Compensation. IEEE Transactions on Neural Systems and Rehabilitation Engineering, 19(2), 177-185 (2011)

11. Derossis, A.M, Fried, G.M., Abrahamowicz, M., Sigman, H.H., Barkun, J.S., Meakins, J.L. : Development of a Model for Training and Evaluation of Laparoscopic Skills. The American Journal of Surgery, 175(6), 482-487 (1998) 